\title{
CICLO VITAL \\ Y ROLES FAMILIARES
}

María Cristina Osorio L.

La ciencia parte de un conjunto de supuestos, entre los cuales, que el mundo es inteligible y sus aspectos físicos, biológicos y sociales son explicables. Es inteligible porque se puede expresar, encontrar y demostrar la existencia de uniformidades; esto supone construir un orden conceptual basado en aquellos aspectos que aparezcan repetitivos e interrelacionados.

¿Es la familia un hecho aislado? ¿Tiene características únicas? Evidentemente no y por esto la Sociología, como otras disciplinas, le destinan parte importante de su estudio.

Al estudiar la familia, desde el punto de vista sociológico, constatamos que su primera uniformidad es la de constituir un grupo social. Como tal, a lo largo de la historia de los pueblos y culturas, asume la responsabilidad de participar activamente en un proceso, por medio del cual el individuo que nace como ser biológico aislado, se transforma en ser social. Ello implica asumir una doble responsabilidad: acoger, cuidar, proteger al nuevo ser $y$, al mismo tiempo, facilitar su proceso de incorporación a la sociedad. Esta "recepción" $y$ "entrega" convierte a la familia en eslabón de enlace entre el individuo y la sociedad, determinando que su función mediatizadora la haga acreedora a la denominación de núcleo básico de la sociedad.

Específicamente la familia, frente al individuo, tiene la compleja tarea de armonizar permanentemente la satisfacción de sus necesidades biológicas, afectivas, materiales, espirituales y sociales, y la de estimular constantemente sus potencialidades para alcanzar el pleno desarrollo de todas ellas.

Frente a la sociedad, la familia debe contribuir a la renovación de sus miembros v a la estabilización de la cultura, transmitiendo e internalizando en sus integrantes os valores e ideas más permanentes, sin descuidar la ejercitación de la comunica- 
ción interpersonal. El cumplimiento de este conjunto de funciones favorecerá la incorporación constructiva de los nuevos miembros, quienes no sólo deben mantener, sino también desarrollar y recrear la vida de la sociedad.

La familia, por lo tanto, como una de las instituciones sociales más estables asegura la mantención y proyección de toda sociedad - no sólo como nexo entre e individuo y la sociedad-sino que, además, se constituye en el punto donde se interconectan las distintas instituciones sociales. Desde esta perspectiva, sirve como el "instrumento" más sensible que tiene toda sociedad para evaluar el efectc de los cambios que ocurran en el plano político, social, económico, ético y cultural.

Una segunda uniformidad, la encontramos en lo que se ha llamado el ciclo vita familiar. Es decir, al igual que todo sistema, ella pasa a través de una serie secuencial de fases a lo largo de su existencia. Estas fases o etapas, a grandes rasgos, se inician con el matrimonio, sigue el nacimiento y crianza de los hijos, el posterior matrimonio y partida de éstos, llamado por algunos: "etapa de nido vacio", en que la pareja vuelve a vivir sola hasta la muerte de uno de sus integrantes.

Íntimamente asociada al ciclo vital familiar, encontramos la tercera uniformidad, ésta dice relación con los roles familiares, que son precisamente los que tipifican y diferencian a la familia de todo otro grupo social. Nuestro modelo familiar actual da origen a 4 pares de roles: esposo - esposa padre - madre / hijo hija / hermano - hermana.

Estas tres uniformidades nos permiten percibir la complejidad y riqueza posible de análisis que ofrece la familia a distintas disciplinas del saber, dándole al mismo tiempo una relevancia tal, que difícilmente se puede pensar en ella como una institución en situación de riesgo, ni menos de extinción. Esta certeza no implica sin embargo, que esté resguardada o exenta de conflictos, crisis o cambios.

La familia como centro del sistema social, consciente o intuitivamente, capta la importancia de su rol social, sintiéndose presionada constantemente para dar satisfacción a las necesidades de sus miembros, al mismo tiempo percibe el control social que la obliga a una adecuación permanente en los estilos de vida que imponen los cambios sociales, todo lo cual le provoca una situación de tensión que genera conflictos. Esta situación constituye a tal extremo la característica genera de la familia de hoy, que amplios sectores de la población estiman que, en relación a otras instituciones o grupos sociales, está en situación deteriorada o crítica.

A su vez, tanto la opinión pública como la literatura sobre el tema, generan confusión al usar como sinónimos los conceptos de "conflicto" y "crisis". Desde la perspectiva sociológica, el conflicto es una condición natural inherente a toda sociedad, razón por la cual distintas corrientes sociológicas se han abocado al estudio de teorías sobre el conflicto, todas ellas coinciden en que el conflicto es un hecho social que se da en toda organización o grupo y que generalmente está fuertemente asociada a los cambios sociales.

Definiremos aqui el conflicto, como un proceso que se manifiesta en desajus- 
tes, ya sea entre los individuos integrantes del grupo familiar, o entre ellos y el medio social; las causas pueden ser endógenas o exógenas y sus efectos pueden ser directa o indirectamente observados.

Vale la pena destacar que el conflicto familiar está normalmente visualizado a nivel intrafamiliar, desconociéndose prácticamente la dimensión macrosocial, es decir la relativa al desajuste que se produce entre la familia y una o varias instituciones sociales.

A modo de ejemplo, señalaremos que en Chile la institución matrimonial entró en conflicto con la institución jurídica al dictarse la ley de matrimonio civil en 1884. Hasta esa fecha, el matrimonio era celebrado y registrado sólo por la Iglesia Católica y el cambio a la autoridad civil produjo una fuerte resistencia, que se hace evidente al observar las tasas de nupcialidad. Estas, desde 1850 hasta hoy han sido extraordinariamente estables (alrededor de 7 a 7,3 por mil), pero tuvieron un brusco descenso a 4,5 por mil en los años posteriores a la dictación de la ley de matrimonio civil; la normalización sólo retornó a mediados de la década de 1920.

De no asociarse el cambio normativo - variable independiente-, a la reducción de las tasas; variable dependiente, se podría pensar que en ese periodo hubo una contracción real y por ende un número significativamente menor de nuevos grupos familiares.

Entendido, pues, el conflicto como un proceso en el que ocurren desajustes de diversa variedad e intensidad, observamos que en todo proceso se registran hitos de intensidad máxima: éstos constituyen las "crisis". En el caso de la familia, la variedad e intensidad de desajustes está condicionado, en general, por variables socioculturales, incluyendo entre éstas las dimensiones políticas y religiosas; por lo tanto, el nivel más alto de desajuste, es también variable. En cualquier caso, el momento en el cual la situación exige tomar una decisión que resuelva o al menos aminore la intensidad del conflicto, es lo que llamamos "crisis".

La crisis familiar es definida por Covarrubias, Muñoz y Reyes, como "el quiebre del equilibrio original de la familia, producido por fallas en sus recursos internos o por circunstancias externas que impiden el adecuado desempeño de las funciones familiares y que implican la búsqueda, por parte de la familia de un nuevo nivel de equilibrio" (1-11).

La crisis, por lo tanto, precede al cambio y hace imperioso buscar nuevas orientaciones o conductas, que atenúen el nivel de tensión $y$, deseablemente, resuelvan el conflicto. Como resultado se producirá un cambio que favorecerá la cohesión del grupo o su ruptura.

La secuencia de "conflictos", "crisis" y "cambios", deja en evidencia que la "crisis" familiar no es un hecho emergente hoy, sino que la encontramos a través de la historia de la humanidad, iniciándose con la expulsión de Adán y Eva del paraíso terrenal; por otra parte, es importante resaltar que las crisis familiares no 
implican necesariamente ruptura ni menos la destrucción de la familia como institución social.

Los conflictos acosan en forma permanente al grupo familiar y algunos conducen a situaciones de crisis bastante generalizadas en cada una de las etapas del ciclo vital familiar; éstos se manifiestan más claramente si se analiza conjuntamente cada etapa del ciclo vital, con los roles sociales que a él corresponden de acuerdo al modelo que cada sociedad se da.

La etapa inicial de formación del matrimonio está precedida por la elección de pareja, decisión que no puede definirse como algo ajeno a la historia de vida y al entorno de cada uno. Esta elección está regulada imperceptiblemente por normas, valores, aspiraciones y modelos que cada uno ha tenido; la elección, por lo tanto, es un proceso y como tal debería ser visualizado no sólo con sentido de presente, sino también proyectado a futuro. ¿Quién soy yo hoy y cómo me veo en 10 ó en 25 años más? ¿Cómo veo hoy y en los años futuros al otro? Estas interrogantes no parecen, sin embargo, ser las que más se estimulan, como parte de la socialización de los jóvenes.

Observamos asimismo, que aún hoy, nuestra sociedad estimula a tomar una decisión "para toda la vida", a una edad relativamente temprana. La edad promedio de matrimonio a nivel nacional, es de 23 años para la mujer y 26 años para el hombre, situación que varía un tanto si se analiza por estratos sociales. Observamos que la mujer de estrato medio-alto y alto tiende a casarse entre los 22 y 24 años, coincidiendo ello con el término de sus estudios superiores y o estabilización laboral, a diferencia de la mujer de estrato bajo que lo hace entre los 16 y 18 años, motivada especialmente por su deseo de independizarse de la familia.

Por otra parte, se estimula la dimensión del amor romántico, que es impulsivo, emocional e irracional, uno y otro se presionan para mantener una relación cerrada, que resulta absorbente y agobiante al largo plazo; no es usual que nuestros modelos socializadores privilegien el desarrollo personal y la madurez favorecida a través de un proceso de estimulación reciproca. El conflicto se produce al intentar armonizar esta situación de "dos en uno", con las presiones sociales hacia la participación de cada uno de ellos en distintos planos de la vida social.

Hay, pues, una alta probabilidad de que se vean enfrentados a una primera crisis, dado que tendrán que compatibilizar su nuevo rol de esposo y esposa, con algunos roles que desarrollaban como solteros; de hecho cada uno pertenece o deseará ingresar a distintos grupos o "comunidades" laborales, estudiantiles, vecinales, religiosas, politicas o deportivas, las que no siempre serán posibles o atrayentes para ambos.

La consolidación de la pareja se favorece, en la medida que ésta tenga la oportunidad de desarrollar espacios de intimidad, de vivir sin presión de tiempo ni interferencia de terceros y de crear particulares formas de comunicación verbal gestural - física y sexual. Esta consolidación permitirá resolver con madurez la crisis 
de roles, sin que ninguno se sienta postergado o limitado por el otro $y$, al mismo tiempo, se avanzará en condiciones de mayor estabilidad hacia la etapa siguiente del ciclo: la llegada del primer hijo. El nacimiento de un hijo, con todas las gratificaciones que se asocian a ello, constituye un hecho que viene a interferir en esta intimidad naciente, la que en muchos casos apenas alcanzó a esbozarse por el breve tiempo transcurrido entre la formalización de la unión y la llegada del hijo. Ser padre o madre no sólo es un nuevo rol que asumir $y$ armonizar con los anteriores, es el único status que, se desee o no, se ejercerá para toda la vida.

El desempeño de este nuevo rol crea tensiones, dada la inseguridad que produce la inexperiencia, al mismo tiempo que favorece los conflictos a nivel de pareja. Ser tres y no dos crea problemas económicos por el gasto que ello involucra, problemas familiares puesto que un niño no sólo da origen al rol de padres, sino también al de abuelos, los que frecuentemente participan con opiniones contrapuestas en normas de crianza, hábitos o pautas de socialización.

Pero sobre todo constituye una limitante a la libertad, razón por la cual si hasta una o dos generaciones atrás la familia se "adaptaba al hijo", hoy es el hijo quien debe hacerlo al estilo de vida de sus padres. Esto implica que los horarios de comidas, de sueño, de silencio, pasen a depender de los espacios de tiempo de los adultos, los que presionados por el mundo externo, intentan combinar el trabajo y a veces los estudios, con la diversión. Hay una clara sobredimensión del mundo adulto-joven que condiciona conductas egoístas, consumistas y más centradas en el presente que en el futuro.

Analizando estos dos pares de roles, de esposos y padres, apreciamos que los cambios económicos - educacionales y sociales han determinado significativas variaciones de forma y de fondo en el desempeño de ellos. Hoy la relación de pareja tiende a ser cada vez más igualitaria, particularmente en los estratos socioeconómicos medios y altos, asociado a niveles más altos de educación, a la necesidad económica de aportar conjuntamente al presupuesto familiar y también, a la dificultad creciente de contar con asesoras del hogar. Sin duda la incorporación de la mujer al trabajo, no sólo le ha dado un grado de mayor seguridad e independencia, sino que además ha obligado a compartir su "reino doméstico" con el esposo; los roles por lo tanto tienden a dejar de ser diferenciales y cada día más son roles intercambiables.

Por otra parte, los niños de todos los estratos ingresan más tempranamente a ardines infantiles, donde no sólo perfeccionan sus hábitos básicos, sino que se hacen más independientes y colaboradores dentro del hogar.

Los cambios antes descritos dicen relación con los roles de cada uno de los ntegrantes del grupo familiar y se dan preferentemente en estructuras familiares nucleares abiertas a compartir la socialización con otras instituciones. El efecto positivo del cambio es la acentuación de conductas autosuficientes y de mayor zreatividad, el riesgo es que la independencia de los integrantes del grupo, debilite 
su identidad y sentido de pertenencia al grupo familiar, acentuando más su integración hacia el medio externo que hacia el enriquecimiento y dinámica interna de la familia.

No puede dejar de considerarse que la familia nuclear tiene como debilidad estructural, el carecer de roles adultos de reemplazo, lo que también incide en la sociedad en que se desarrollan los niños y se apagan los ancianos.

El opuesto a esto se encuentra con frecuencia en estructuras familiares extendidas, más cerradas en sí mismas, con limitadas relaciones hacia el mundo externo llegando al extremo de constituirse en pequeñas unidades de producción y consumo. La gran ventaja aquí es la estabilidad como grupo, el apoyo afectivo de sus miembros y la seguridad de ofrecer a los menores los roles de adulto de padre y madre, puesto que aún en el caso de faltar alguno por muerte o separación, otros miembros adultos (abuelos o tíos) asumirán el reemplazo; el rol del género está por lo tanto claramente perfilado.

Una alta proporción de las crisis en la etapa de expansión de la familia ocurre a nivel de pareja en relación al tamaño y distanciamiento entre los hijos. Los valores religiosos, las presiones económicas y sociales afloran con gran fuerza, tanto para robustecer la unión cuando éstos son compartidos, como para debilitarla hasta la ruptura, cuando la unión se ha sostenido sobre bases de comunicación superficialy conductas orientadas por pautas disímiles o peor aún, antagónicas.

Al respecto, un estudio sobre ruptura matrimonial indica que el $34 \%$ de las parejas se separaron después de 1 a 4 años de matrimonio y el $45,5 \%$ de ellas transcurridos 5 a 9 años (2-91). Si esto lo relacionamos con las tasas de natalidad promedio del país que arrojan, como moda estadistica, familias con 2 hijos, la ruptura ha ocurrido preferentemente cuando la etapa de expansión ha dado paso a la estabilización en términos del ciclo vital familiar. Estas mismas parejas se habían formado, en el 57\% de los casos, antes de cumplir la mujer 21 años (2-14), es decir en una etapa en que los proyectos de vida $y$ los valores no están al menos conscientemente asentados.

La etapa de estabilización se centra en la socialización de los hijos y las familias se van perfilando en cuanto a las conductas que priman en la relación de los adultos, como especialmente en la de éstos hacia sus hijos. La literatura tipifica tres "modelos" de padres: a) permisivos; b) autoritarios y c) autorizadores.

Los "permisivos", en términos generales, son aquellos que intentando estimular el autocontrol y autorrealización del niño, regulan escasamente los controles y patrones normativos consultando su opinión a los hijos en todas las decisiones familiares.

Los "autoritarios", por el contrario, mantienen la estabilidad y orden del grupo bajo un fuerte control, quien posee la autoridad pocas veces consulta al resto y hace de la obediencia un valor.

Finalmente, los padres "autorizadores" mantienen una orientación firme, cons- 
tante y racional, que estimula la independencia dentro de los marcos normativos; los valores centrales aquí son la responsabilidad y el compromiso.

Estos tres tipos de modelos socializadores marcan no sólo la dinámica interna del grupo familiar durante la etapa de consolidación o estabilidad del ciclo, sino que se proyecta a las etapas siguientes de desmembramiento y término del ciclo.

El poder y prestigio de los padres permisivos es muy bajo entre los adolescentes, quienes buscan modelos fuera del hogar y por ende al elegir pareja, es altamente probable que ésta sea para los padres inexplicablemente diferente a sus expectativas; es posible incluso que de elegir como pareja a alguien socializado en alguno de los otros modelos, se integre mejor a sus suegros que a sus propios padres.

Los hijos de padres autoritarios tienden a tener una clara concepción del sistema social y del desempeño de roles dentro de él, originando, según el clásico estudio de Adorno "La Personalidad Autoritaria", preferentemente dos tipos de conductas: sometidas o rebeldes. Los primeros serán inseguros y dependientes e intentarán revivir en su nueva familia el mismo esquema de sus padres, permaneciendo por largo tiempo con una fuerte dependencia hacia ellos. Los segundos en cambio buscarán lo opuesto y es altamente probable que se casen tempranamente como una vía de escape a la autoridad paterna.

Los hijos de padres autorizadores, formados para madurar evaluando sus éxitos y fracasos, tenderán a ser más creativos, seguros e independientes manteniendo lazos estrechos con sus padres, pero restringiendo la participación de sus familias de orientación (los padres), en su propia familia.

Las crisis al interior de las nuevas familias estarán fuertemente condicionadas por los modelos socializadores de cada uno. Por su parte, los padres en esta etapa de contracción del ciclo, asumirán dos nuevos pares de roles el de suegros y el de abuelos. La satisfacción o frustración que experimenten en cada uno de ellos estará sin duda muy condicionada al modelo socializador que tipifica la familia en las etapas precedentes del ciclo.

Finalmente, el reencuentro de la pareja hace de éste un período que puede llegar a ser el más largo del ciclo familiar. A la edad de 30 años, en promedio, la mujer ha terminado su ciclo reproductivo y por ende entre las edades de 45 y 55 años ha quedado el "nido vacio". Los 15-20 ó más años de vida conyugal posterior, liberados de la presión y responsabilidad de socializar a los hijos, también estarán marcados por la capacidad y entrenamiento ya alcanzados como pareja para compartir o no las distintas manifestaciones de la cultura y las relaciones interpersonales, vivencias religiosas e ideológicas, satisfacción de necesidades materiales y enfrentar en forma conjunta el deterioro de la salud física y mental que inevitablemente ocurrirá. Hoy que la esperanza de vida se amplía y este problema se agudiza.

La etapa final del ciclo plantea a la pareja la obligación imperiosa de encararse a sí mismo y al otro, con quien se ha compartido o caminado en vías paralelas por tantos años; ya no hay presencia de los hijos para centrar la atención en ellos; en 
este periodo ocurre mayoritariamente el término de la etapa activa laboral, dando ambos hechos paso a gran cantidad de espacios de libertad. El conflicto y las crisis aquí se derivan del uso del tiempo libre. La presencia permanente del hombre, que siempre estuvo fuera del hogar, viene a ser con frecuencia un elemento perturbador.

Si en esta época se intenta mantener la división de roles, puesto que ya resulta difícil cambiar, el hombre se sentirá inútil dentro de su propia casa y si no cuenta con recursos económicos suficientes, o una cultura que le permita disfrutar de los diversos ámbitos del saber científico-tecnológico o artístico, su deterioro físico y mental se acelerará. Es difícil que las parejas que no desarrollaron la habilidad de comunicarse la descubran ahora, acentuarán más bien su incomunicación, aislándose no sólo entre sí mismos, sino también hacia el exterior. Quienes, en cambio, compartieron con plenitud los altos y bajos familiares, encontrarán en ésta una etapa grata que para muchos podrá ser la de sus "años dorados".

\section{CITAS BIBLIOGRÁFICAS}

Covarrubias, Paz; Muñoz, Mónica; Reyes, CarMEN. Crisis en la Familia. Cuadernos del Instituto de Sociología. Universidad Católica, 1983
Parada, Germán. Separación Matrimonial. Tesis de Grado para obtener Titulo de Licenciado en Sociología, U. de Chile, 1983. 